\title{
"Investigation of the Periodontal and Microbiological Status of Patients Undergoing Fixed Orthodontic Therapy"
}

\author{
Dr. Verdine Virginia Antony, Dr. Rahamathulla Khan \\ ${ }^{1}$ ( Department of Periodontics \& Implantology, Sirte University, Libya.) \\ 2 ( Department of Orthodontics, Sirte University, Libya.)
}

\begin{abstract}
The aim of the study was to investigate the periodontal and microbiological status of patient undergoing fixed orthodontic treatment.

Methods: In this study, plaque samples were collected from 12 patients at baseline, four monthly visits during orthodontic treatment and at 30 days after removal of the appliance. A benzoyl-DL-arginine-naphthylamide (BANA) test was performed to identify the periodontal pathogens. Dark field microscopy was used to recognize the morphotypes. The Plaque Index and probing depths were assessed at each test interval to determine the hygiene and periodontal status of the patients. Data were analyzed using analysis of variance and Tukey's test. Results: Significant increase in plaque score, probing depths, and BANA scores were found at each interval after placement of orthodontic appliances. The levels, however returned to baseline after removal of the appliances. Dark field microscopy confirmed increases in small spirochetes (8.5\%), large spirochetes (2\%), non-motile rods (8.5\%), fusiforms (5.5\%), and filaments (1\%) with orthodontic treatment.

Conclusions: Patients undergoing orthodontic therapy have an increase in plaque accumulation, probing depth, and microbial activity that may be associated with periodontal destruction. Thirty days after removal of the orthodontic appliance, the plaque score, probing depth, and BANA test score returned to almost baseline level.
\end{abstract}

Key words: orthodontic appliance, plaque index, probing depth.

\section{Introduction}

The relationship between orthodontic procedures and periodontal status is considered a challenge, especially periodontal health during and after orthodontic treatment. It is well established that the patients who undergo orthodontic treatment have a high susceptibility to present plaque accumulation on their teeth because of the presence of bands, brackets, wires and other orthodontic attachments which impair plaque removal, proper oral hygiene, and gingival health [1]. This promotes specific alteration in the oral environment, including decreased $\mathrm{pH}$, increased plaque accumulation[2], and elevation of microbial counts in the saliva and the biofilm [3] Orthodontic treatment is a double-action procedure, regarding the periodontal tissues, which may be sometimes very significant in increasing the periodontal health status, and sometimes a harmful procedure which can be followed by several types of periodontal complications, namely: gingival recessions, bone dehiscence, gingival invaginations and/or the formation of gingival pockets. Recent studies show that the most important etiological factor of periodontal disease is plaque deposition around gingival margin[2,3].

Three periodontal pathogens that are known to inhabit the plaque and contribute to periodontal disease are Porphyromonas gingivalis, Treponema denticola, and Tannerella forsythia [4]. These bacteria are anaerobic periodontal pathogens capable of initiating periodontal destruction. In many studies, the presence of these bacteria has been correlated with the common forms of adult periodontitis [5,6]. Although most patients undergoing orthodontic therapy tend to be younger and are less likely to experience periodontal disease, the host resistance to bacteria is compromised in orthodontic patients due to the appliances on the teeth. These bacteria are termed the 'red complex' among five bacterial complexes that group bacteria together based on relationships and associations. These three bacteria have a symbiotic relationship in a highly ordered system or biofilm, which serves as protection, facilitates communication, and promotes adhesion to the oral environment [6].

The benzoyl-DL-arginine-naphthylamide (BANA) test (Knowell Periodontal Technologies, Toronto, Canada) has been used by many investigators to rapidly screen contributing putative red complex periodontal pathogens in dental patients $[4,7]$. The BANA test is accurate and efficient in determining whether the red complex bacteria are present in a plaque sample [4,7]. Patients who demonstrate susceptibility to these bacteria can be identified and monitored for increases in the bacterial load with the BANA test. To complement data obtained from the BANA test, dark field microscopy was used to ascertain the morphotypes of periodontal pathogens residing in the plaque. The purpose of this study was to determine the BANA-positive periodontal pathogens and periodontal status in patients undergoing fixed orthodontic treatment. 


\section{Materials And Methods:}

Twelve patients undergoing fixed orthodontic treatment, with a mean age of $16( \pm 2)$ years, were selected for the study.

\section{Inclusion criteria:}

- Presence of full adult dentition,

- Orthodontic treatment with fixed appliances (metal brackets) in the upper and lower arches (archwire sequence included 0.012-inch nickel titanium [NiTi], 0.018-inch NiTi, 0.018-inch stainless steel [SS], and $0.018 \times 0.025$-inch SS wires)

- No pre-existing periodontal disease

- No antibiotic treatment in the previous 14 days

- Availabile for data collection during the first 4 months of orthodontic treatment (i.e $2^{\text {nd }}$ and $5^{\text {th }}$ visits) and one recall visit 30 days after completion of orthodontic treatment.

Approval from the ethical committee was procedure and informed consent was obtained from all patients. Standard oral hygiene instructions and oral hygiene aids were given at the beginning of orthodontic treatment.

\section{1 MICROBIAL ANALYSIS:}

Prior to placement of the orthodontic appliances, a plaque score was recorded, probing depths were measured, and five plaque samples were taken for laboratory analysis. Four Stim-U-Dent interdental cleaners (Johnson and Johnson, USA) were used for collection of the plaque samples. For consistency, plaque samples were collected from each quadrant from the interproximal area between the first molars and second premolars by one operator. The samples were taken with the tip of a Stim-U-Dent, placed slightly subgingivally, with a scraping motion. Samples were transferred to the BANA Reagent Strips (USA). The BANA reagent (USA) and strips were plastic cards onto which reagent matrices were affixed. The Stim-U Dent interdental cleaners with the plaque were scraped onto the inferior reagent matrix with linear scribes. This was done to provide a maximum length of plaque to evaluate the BANA reaction. The inferior reagent matrix, to which subgingival plaque samples were added, was impregnated with buffered N-BANA. The superior reagent matrix was impregnated with stabilized Evans black dye (chromogenic diazo reagent). The reaction was activated when the inferior strip was folded over the superior strip, which had been moistened with distilled water, thus enabling any naphthylamide liberated from the inferior reagent matrix (BANA impregnated strip) to diffuse into the superior strip. Naphthylamide could then react with the Evans black dye, yielding a permanent blue-black color spot on a pale reddish-brown background. An incubation period of 15 minutes at $55^{\circ} \mathrm{C}$ was used to increase the sensitivity of the Perioscan card. After incubation, the reagent cards were stored in sealed light-protected envelopes and analyzed at the end of the regular orthodontic appointment by the investigators. The results were examined 24 hours later.

The Perioscan reagent test criteria were read as follows:

1) No color observable on the background signifies a titer of periodontal pathogens of insignificant numbers ( $<10000$ colony-forming units [CFU]) or a negative recording;

2) A small, faint blue on the background signifies a titer of detectable numbers of periodontal pathogens of some clinical significance (10 000-99 $999 \mathrm{CFU}$ ) or a weak positive recording; and

3) A distinct and darker blue color on the background signifies a substantial and clinically relevant titer ( $\geq 100000 \mathrm{CFU}$ ) of pathogens present in the plaque sample or a positive recording.

Data of the above three categories were recorded as negative, weak positive or positive respectively.

For dark field microscopy, a plaque sample was taken from the lower right canine/premolar area with a StimUDent and placed in a stored liquid dental transport medium. A Stim-U-Dent was used to stir the solution and the vial was closed and sent to the Department of Pathology. The dark field microscopic analysis consisted of a quantification and qualification of the nine periodontal pathogenic morphotypes. The nine categories of qualification were as follows: small spirochetes, intermediate spirochetes, large spirochetes, motile rods, coccoid forms, non-motile rods, fusiforms, filaments, and yeast. The investigator qualified and quantified the organisms based on their shape. Proportions were calculated for each of the organisms relative to the 100 total counts. These measurements served as the baseline (i.e $1^{\text {st }}$ visit) against which the BANA scores were compared. These procedures were carried out at each of the next 5 visits, for a total of 6 recordings.

\section{II.2 PLAQUE SCORE AND PROBING POCKET DEPTH}

After the plaque samples were taken for the BANA test, probing depths of selected teeth and plaque scores were measured by one operator. Probing depths were measured with a Michigan periodontal probe (HuFriedy, USA) and taken on the mesial, middle, and distal sides of the facial and lingual surfaces of the upper and lower first molars and second premolars (first premolars were used when second premolars were not present). Thus, 12 surfaces were probed in each of the four quadrants. The plaque score was then assessed using the O'Leary Plaque Index[8] whereby all teeth were disclosed with plaque-disclosing solution and each tooth had a 
mesial, distal, facial, and lingual surface scored for plaque. The plaque score was calculated by dividing the number of plaque surfaces by the total number of tooth surfaces. At each subsequent appointment, plaque samples were taken for BANA test and dark field microscopic analysis, and the plaque scores and probing depths measured in the same manner.

\section{II.3 DATA ANALYSIS}

Plaque scores were measured and recorded as percentages. Probing depths were measured and recorded as millimeters (mm). The BANA test results measured as negative, weak positive, or positive were converted to 0,1 , and 2, respectively for data analysis. Plaque scores, probing depths, and BANA scores were consolidated and averaged for all patients at each visit to determine the variations between patients and time intervals. Means, SDs, and relative frequencies were tabulated. Analysis of variance (ANOVA) was used to analyze data, with a P value of $\leq 0.05$ regarded as statistical significance. A Tukey's test was used to examine differences between patient visits or months.

\section{Results}

The ANOVA test revealed significant differences between patient visits $(\mathrm{P}<0.001)$. The Tukey's test revealed significantly higher mean $( \pm \mathrm{SD})$ plaque scores for the subsequent four visits (visits 2-5) during orthodontic treatment $(48.90 \pm 143.05 \% ; 51.27 \pm 13.25 \% ; 52.65 \pm 14.86 \% ; 54.55 \pm 18.90 \%)$ compared with baseline $(39.25 \pm 15.85 \%)[\mathrm{P}<0.05]$. No significant differences were found between the plaque score obtained 30 days after removal of the orthodontic appliance $(45.55 \pm 14.80 \%)$ and baseline. The individual O'Leary Plaque Index scores for each visit for all 12 patients are shown in TABLE 1. The ANOVA test revealed significant differences among patients $(\mathrm{P}<0.001)$, indicating variable differences between plaque scores both within and between patients. The ANOVA test revealed significant differences between the 6 visits at $P<0.001$. The Tukey's HSD test revealed significantly higher mean $( \pm \mathrm{SD})$ probing depths for visit $3(2.70 \pm 0.57 \mathrm{~mm} ; \mathrm{P}<0.05)$, visit $4(2.85 \pm 0.25 \mathrm{~mm} ; \mathrm{P}<0.05)$, and visit $5(2.09 \pm 0.78 \mathrm{~mm} ; \mathrm{P}<0.05)$ than that for the baseline $(2.35 \pm 0.55 \mathrm{~mm})$. No significant differences were found between visit $2(2.50 \pm 0.59 \mathrm{~mm})$ and 30 days after removal of the appliances $(2.54 \pm 0.54 \mathrm{~mm})$. The probing depth averages for patients ranged from $2.3 \mathrm{~mm}$ to $3.2 \mathrm{~mm}$. The individual probing depths for all 12 patients at each visit are shown in Table 2. The ANOVA test did not reveal significant differences among patients $(\mathrm{P}=0.08)$.

\section{1 BANA test}

The ANOVA test revealed significant differences among the six time intervals $(\mathrm{P}<0.001)$. The Tukey's test revealed significantly higher mean $( \pm \mathrm{SD})$ BANA test scores during orthodontic treatment for visit 2 $(0.65 \pm 0.48)$, visit $3(1.00 \pm 0.51)$, visit $4(1.52 \pm 0.57)$, and visit $5(1.41 \pm 0.50)$ compared with baseline $(0.34 \pm 0.51)$ and 30 days after removal of the appliances $(0.35 \pm 0.40) \quad[\mathrm{P}<0.05]$. The individual BANA test scores for each visit are shown in Table 3. The ANOVA test revealed significant differences among patients $(\mathrm{P}<0.001)$, indicating variability among patients.

\section{2 Dark field microscopy}

Dark field microscopy was performed on plaque samples, and nine morphotypes were evaluated. The count for small spirochetes showed a steady increase in the numbers and relative percentages during orthodontic treatment from $2.3 \%$ at the baseline to $9.8 \%$ for visit 5, representing a $8.0 \%$ increase in total organisms. The count for intermediate spirochetes showed an increase from $8.4 \%$ at baseline to $10 \%$ in visit 3 and declined in visits 4 and 5 down to $6.9 \%$. The count for large spirochetes showed a $2.0 \%$ increase from $8.9 \%$ at the baseline to $12.10 \%$ in visit 5 . The count for motile rods showed a steady decrease from $18 \%$ at baseline to $10 \%$ in visit 5 . The count for coccoid forms showed a large decrease from $30 \%$ at baseline to $10 \%$ in visit 5 . The count for nonmotile rods showed a steady $8.5 \%$ increase from $24 \%$ at baseline to $31 \%$ in visit 5 . The count for fusiforms showed a slight but steady increase from 5.5\% at baseline to $11 \%$ in visit 5 . The count for filaments showed only a small increase of $1 \%$ from $5 \%$ at baseline to $6 \%$ in visit 5 . The count for yeasts increased from $1 \%$ at baseline to $3 \%$ in visit 3 , and decreased to $2 \%$ in visit 5 .

\section{Discussion:}

This study examined the periodontal and microbiological status of patients undergoing orthodontic therapy. The main aim of the study was to evaluate the microbiological status, along with it the plaque scores and probing depths were also evaluated to assess the periodontal health of the patients. Several studies have investigated the effects of orthodontic treatment on periodontal health $[9,10]$. Most of the investigators concluded that the overall gingival changes produced by appliances are transient with no permanent damage to the periodontal tissues. The current study found that the plaque scores increased over time in patients undergoing orthodontic therapy. The increase in plaque scores on teeth in this study is in agreement with several 
other studies[10,11].The current study found that the greatest increase in plaque score occurred immediately after placement of orthodontic appliances, with an overall increase of $18.3 \%$. Individual variations in plaque scores were also observed. Patients exhibited the same plaque score at baseline and visit 5 . When proper oral hygiene measures are instituted in patients undergoing orthodontic therapy, optimal hygiene and plaque control can be achieved $[12,13]$. This study also found an increase in mean probing depth with orthodontic treatment. The differences were generally small, but significant differences were found between visits 3,4 , and 5 from the baseline. The probing depths had more of a uniform increase than the plaque scores after the placement of orthodontic appliances. The probing depth increases found in this study were small, but significant. A common clinical sequel of orthodontic therapy is gingival hyperplasia. This is a result of the inflammatory process and plaque accumulation, and will cause the probing depth to increase, especially in the papillary areas. Another possible reason for increased probing depth in patients undergoing orthodontic therapy is the increased risk for penetration of the periodontal probe through the junctional epithelium in inflamed gingiva [14].This study found an increase in BANA scores with orthodontic treatment. The scores at visits 3 to 5 were significantly greater than that at the baseline, showing the largest increase at 4 months after the orthodontic appliances were placed. The BANA test (Perioscan) has been shown to be a reliable indicator for the presence of the three putative periodontal pathogens (red complex): $P$ gingivalis, $T$ denticola, and $T$ forsythia $[3,7,8]$. These bacteria have been correlated with the common forms of adult periodontitis. This study involved 8 adults and 4 adolescents, and that these bacteria were found in the plaque samples of the latter, indicating that these three periodontal pathogens are found not only in adults, but also in adolescents $[3,15]$. The BANA test indicated the presence of the three periodontal pathogens in all patients, with variations in levels detected. The results showed statistically significant differences in BANA scores over time in both adult and adolescent patients undergoing orthodontic therapy. The results of dark field microscopy gave a detailed picture of the microbial populations in the orthodontic plaque samples. The sampling in this study consisted of nine morphotypes of common oral microbes. The life cycle of bacterial plaque has been established by many authors [15]. The early colonizers of the biofilm are mostly Gram-positive cocci and rods. These bacteria colonize on clean tooth surfaces with adhesins and they help to form the framework for the secondary colonizers, which colonize by coagregation. The secondary bacteria may be Gram negative, and have a preponderance of rods, fusiforms, cocci, filaments, and spirochetes. These bacteria include $P$ gingivalis, $T$ forsythia, and $T$ denticola, and it is these organisms that cause concern. This study found statistically significant increase from baseline in the following morphotypes: small spirochetes, large spirochetes, non-motile rods, fusiforms, and filaments. The largest increase in population was seen in the small spirochetes and non-motile rods. In the present study, a decrease in all coccoid forms was found. The results of this study are also comparable with previous studies on the progression of gingivitis and its microbial association.[16]. The correlation between microscopy and BANA is not entirely coherent. Treponema denticola is a spirochete, and microscopy found a considerable increase in small spirochetes than large spirochetes . However, $T$ forsythia and $P$ gingivalis are Gram-negative rods, and this study found an increase in motile rods.

This study also found a return to almost baseline level of plaque score, probing depth, and BANA score 30 days after removal of the orthodontic appliances. Clinically, the degree of gingival inflammation usually improves after removal of the appliances [15]. Some studies on the activity of the periodontal pathogenic bacteria, after removal of orthodontic appliances, showed that the presence of these bacteria during treatment does not signify active periodontal disease. However, the presence of these bacteria may predispose the patient to periodontal disease. In addition, when host immune- resistance or equilibrium (locally and systemically) is altered or challenged, these bacteria may initiate periodontal disease. The biofilm may become unstable, allowing the red complex bacteria to play an active role in periodontal destruction. The high BANA score averages may be explained by the longer incubation time used (15 minutes instead of 5 minutes). This study used 15 minutes for incubation because it is believed that the 5-minute incubation period may not result in detectable levels of bacteria. The increase in BANA scores is an important aspect of this study, because the operator consistently found higher bacterial titers over time.

Plaque is the primary etiological agent in almost all periodontal and gingival conditions. Thus, plaque control must be emphasized as the most important factor in preserving periodontal health in patients undergoing orthodontic therapy. Orthodontic appliances present a challenge to the proper removal of plaque from the tooth and gingival surfaces. The plaque may exist in equilibrium with the patient in the oral environment. However, this equilibrium may become unstable over time and with alterations in the external environment. Organisms commonly present in an early plaque are Gram-positive rods and cocci. Over time, these organisms are replaced by more Gram-negative and anaerobic organisms, which may initiate a periodontal reaction. The biofilm may become unbalanced and pathologic as a result of changes in the environment from orthodontic appliances. The orthodontic appliances cause mechanical plaque traps where plaques may evolve into a pathological state, because adequate oral hygiene measures are more difficult to achieve during orthodontic therapy. Thus, patient motivation and oral hygiene education are essential elements to a successful orthodontic treatment outcome. 


\section{TABLES}

TABLE 1: Mean plaque score for each patient at each visit

\begin{tabular}{|c|c|c|c|c|c|}
\hline \multirow{2}{*}{ Patient No } & \multicolumn{5}{|c|}{ PLAQUE SCORE (\%) } \\
\cline { 2 - 5 } & $\begin{array}{c}\text { Visit 1 } \\
\text { (Baseline) }\end{array}$ & Visit 2 & Visit 3 & Visit 4 & Visit 5 \\
\hline 1. & 23 & 34 & 40 & 42 & 47 \\
\hline 2. & 47 & 53 & 52 & 55 & 54 \\
\hline 3. & 37 & 40 & 48 & 50 & 52 \\
\hline 4. & 27 & 42 & 46 & 51 & 60 \\
\hline 5. & 42 & 53 & 57 & 56 & 63 \\
\hline 6. & 63 & 64 & 66 & 57 & 63 \\
\hline 7. & 27 & 43 & 52 & 54 & 58 \\
\hline 8. & 40 & 47 & 56 & 61 & 68 \\
\hline 9. & 43 & 50 & 57 & 62 & 70 \\
\hline 10. & 17 & 25 & 38 & 43 & 50 \\
\hline 11. & 57 & 60 & 68 & 69 & 73 \\
\hline 12. & 50 & 57 & 60 & 64 & 70 \\
\hline
\end{tabular}

TABLE 2: Mean probing depth for each patient at each visit

\begin{tabular}{|c|c|c|c|c|c|}
\hline \multirow{2}{*}{ Patient No } & \multicolumn{5}{|c|}{ PROBING DEPTH (mm) } \\
\cline { 2 - 6 } & $\begin{array}{c}\text { Visit 1 } \\
\text { (Baseline) }\end{array}$ & Visit 2 & Visit 3 & Visit 4 & Visit 5 \\
\hline 1. & 2.70 & 2.73 & 2.75 & 2.80 & 3.00 \\
\hline 2. & 2.60 & 2.70 & 2.73 & 2.75 & 2.85 \\
\hline 3. & 2.19 & 2.60 & 2.73 & 2.80 & 2.90 \\
\hline 4. & 2.42 & 2.60 & 2.70 & 2.75 & 2.80 \\
\hline 5. & 2.25 & 2.54 & 2.65 & 2.75 & 2.95 \\
\hline 6. & 2.56 & 2.60 & 2.60 & 2.73 & 2.85 \\
\hline 7. & 2.25 & 2.50 & 2.60 & 2.70 & 2.85 \\
\hline 8. & 2.50 & 2.63 & 2.70 & 2.80 & 3.20 \\
\hline 9. & 2.40 & 2.56 & 2.60 & 2.74 & 3.00 \\
\hline 10. & 2.45 & 2.68 & 2.70 & 2.85 & 2.90 \\
\hline 11. & 2.40 & 2.67 & 2.74 & 2.90 & 3.10 \\
\hline 12. & 2.60 & 2.70 & 2.71 & 2.80 & 3.00 \\
\hline
\end{tabular}

TABLE 3: Mean BANA Test score for each patient at each visit

\begin{tabular}{|c|c|c|c|c|c|}
\hline \multirow{2}{*}{ Patient No } & \multicolumn{5}{|c|}{ PROBING DEPTH (mm) } \\
\cline { 2 - 6 } & $\begin{array}{c}\text { Visit 1 } \\
\text { (Baseline) }\end{array}$ & Visit 2 & Visit 3 & Visit 4 & \\
\hline 1. & 0.75 & 1.00 & 1.00 & 1.25 & 1.25 \\
\hline 2. & 0.50 & 1.00 & 1.25 & 1.25 & 1.75 \\
\hline 3. & 0 & 0 & 0.50 & 1.25 & 1.50 \\
\hline 4. & 0.50 & 1.00 & 1.25 & 1.25 & 1.25 \\
\hline 5. & 0.50 & 0 & 0.50 & 0.50 & 0.50 \\
\hline 6. & 1.00 & 1.00 & 1.00 & 1.25 & 1.25 \\
\hline 7. & 0 & 0.25 & 0.25 & 1.00 & 1.00 \\
\hline 8. & 0.75 & 0.75 & 0.50 & 0.75 & 1.00 \\
\hline 9. & 0.50 & 1.00 & 1.00 & 1.50 & 1.25 \\
\hline 10. & 0.50 & 0.50 & 1.25 & 1.25 & 1.50 \\
\hline 11. & 0 & 0 & 0.25 & 1.00 & 1.75 \\
\hline 12. & 1.00 & 1.00 & 1.50 & 1.50 & \\
\hline
\end{tabular}

\section{Conclusions:}

Plaque score and probing depths increased with successive orthodontic visits after placement of orthodontic appliances. There was a concurrent increase in the BANA positive periodontal pathogens $P$ gingivalis, $T$ denticola, and $T$ forsythia with orthodontic treatment. Dark field microscopy confirmed an increase in the populations of small spirochetes, large spirochetes, non-motile rods, filaments and fusiforms, as well as a decrease in the populations of all coccoid forms and motile rods during orthodontic therapy. Thirty days after removal of the orthodontic appliance, the plaque score, probing depths and BANA score returned to almost baseline levels. 


\section{References}

[1]. Naranjo AA, Trivino ML, Jaramillo A, Betancourth M, Botero JE. Changes in the subgingival microbiota and periodontal parameters before and 3 months after bracket placement. Am J Orthod Dentofacial Orthop 2006;130:275.17-22.

[2]. Ai H, Lu HF, Liang HY, Wu J, Li RL, Liu GP, Xi Y. Influences of bracket bonding on mutans streptococcus in plaque detected by real time fluorescence-quantitative polymerase chain reaction. Chin Med J (Engl) 2005;118:2005-10.

[3]. Hirsch DI, Kulbersh R, Kaczynski R. Assessment of pretreatment orthodontic patients using the BANA test. N-benzoylDLarginine- naphthylamide. Am J Orthod Dentofacial Orthop 1997;112:154-8.

[4]. Atack NE, Sandy JR, Addy M. Periodontal and microbiological changes associated with the placement of orthodontic appliances. A review. J Periodontol 1996;67:78-85.

[5]. Socransky SS, Haffajee AD, Cugini MA, Smith C, Kent RL Jr. Microbial complexes in subgingival plaque. J Clin Periodontol 1998;25:134-44.

[6]. Loesche WJ, Lopatin DE, Giordano J, Alcoforado G, Hujoel P. Comparison of the benzoyl-DL-arginine-naphthylamide (BANA) test, DNA probes, and immunological reagents for ability to detect anaerobic periodontal infections due to Porphyromonas gingivalis, Treponema denticola, and Bacteroides forsythus. J Clin Microbiol 1992;30:427-33.

[7]. Loesche WJ, Giordano J, Hujoel PP. The utility of the BANA test for monitoring anaerobic infections due to spirochetes (Treponema denticola) in periodontal disease. J Dent Res 1990;69:1696-702.

[8]. O'Leary TJ, Drake RB, Naylor JE. The plaque control record. J. Periodontol 1972;43:38

[9]. Sadowsky C, BeGole EA. Long-term effects of orthodontic treatment on periodontal health. Am J Orthod 1981;80:156-72.

[10]. Polson AM, Subtelny JD, Meitner SW, et al. Long-term periodontal status after orthodontic treatment. Am J Orthod Dentofacial Orthop 1988;93:51-8.

[11]. Speer C, Pelz K, Hopfenmüller W, Holtgrave EA. Investigations on the influencing of the subgingival microflora in chronic periodontitis. A study in adult patients during fixed appliance therapy [English and German]. J Orofac Orthop 2004;65:34-47.

[12]. Orrico SR, de Toledo BE, Fonseca L, Ito IY. Influence of age on the reactivity of the BANA test among Brazilian children. Bra z Dent J 1991;2:115-22.

[13]. Watson MR, Lopatin DE, Bretz WA, Ertel IJ, Loesche WJ. Detection of two anaerobic periodontal pathogens in children by means of the BANA and ELISA assays. J Dent Res 1991;70:1052-6.

[14]. Watson MR, Bretz WA, Loesche WJ. Presence of Treponema denticola and Porphyromonas gingivalis in children correlated with periodontal disease of their parents. J Dent Res 1994;73:1636-40.

[15]. Socransky SS, Smith C, Martin L, Paster BJ, Dewhirst FE, Levin AE. "Checkerboard" DNA-DNA hybridization. Biotechniques 1994;17:788-92.

[16]. Andrucioli MC, Nelson-Filho P, Matsumoto MA, et al. Molecular detection of in-vivo microbial contamination of metallic orthodontic brackets by checkerboard DNA-DNA hybridization. Am J Orthod Dentofacial Orthop 2012;141:24-9. 